\title{
Magnetic anisotropy and magnetic excitations in supported atoms
}

\author{
Tobias Schuh, ${ }^{1}$ Timofey Balashov, ${ }^{2}$ Toshio Miyamachi, ${ }^{1}$ Shih-Yu Wu, ${ }^{3}$ Chein-Cheng Kuo, ${ }^{3}$ \\ Arthur Ernst, ${ }^{4}$ Jürgen Henk, ${ }^{4}$ and Wulf Wulfhekel ${ }^{1}$ \\ ${ }^{1}$ Karlsruhe Institute of Technology, Physikalisches Institut, Wolfgang-Gaede Str. 1, D-76131 Karlsruhe, Germany \\ ${ }^{2}$ Centre d'Investigacions en Nanociència i Nanotecnologia (ICN-CSIC), E-08193 Barcelona, Spain \\ ${ }^{3}$ Department of Physics, National Sun Yat-sen University, Kaohsiung 80424, Taiwan \\ ${ }^{4}$ Max-Planck Institut für Mikrostrukturphysik, Weinberg 2, D-06120 Halle, Germany
}

(Received 29 April 2011; revised manuscript received 19 July 2011; published 1 September 2011)

\begin{abstract}
We present a view on inelastic scanning tunneling spectroscopy of magnetic impurities relying on states of the total angular momentum $J=L+S$ in the presence of a crystal field. We show that the selection rules for spin-flip scattering within the $J$-multiplet agree with the simple selection rules for the effective spin model, but also show the deviations from the latter for the transition probabilities. A reinterpretation of some recent experimental findings in a description based on the total angular momentum $J$ is done.
\end{abstract}

\section{INTRODUCTION}

Recent investigations of magnetic atoms and nanoclusters $^{1-5}$ show potential pitfalls in understanding nanomagnetism on a quantitative level. In experiments using spin-flip scattering, one often uses an effective spin model, $, 6,7$ which expresses the magnetic state of an atom or a cluster in terms of an effective quantum spin $\tilde{S}$, i. e., by the eigenvalues of the operators $\tilde{S}^{2}$ and $\tilde{S}_{z}$. This approximation has been widely used predominantly in the field of molecular magnets. ${ }^{8,9}$ Typically, the effective spin $\tilde{S}$ is assumed to be of identical size as the real $S$ of the magnetic atom. This is justified provided that the crystal field completely quenches the orbital momentum $L$. The orbital momentum is often, however, not quenched completely, as x-ray magnetic circular dichroism (XMCD) measurements have shown, e. g., in the case of Co atoms adsorbed on $\operatorname{Pt}(111)$ (Ref. 4) and has to be considered in the effective spin model. The observed large magnetic anisotropy energy (MAE) is the energy to turn the magnetization $\boldsymbol{M}$. Its origin is a strong spin-orbit interaction that couples the spin $S$ and the orbital momentum $\boldsymbol{L}$ of the atoms to the total angular momentum $\boldsymbol{J}$. Further, the crystal field ties the orbital momentum to the crystallographic directions of the atom's surroundings. These interactions produce energetically favorable directions for the magnetization $\boldsymbol{M}$ and imply a nonzero MAE.

The effective spin model has been used for spin-flip experiments, in particular, in inelastic tunneling spectroscopy ${ }^{2,3}$ (ITS) on $\mathrm{Co}, \mathrm{Fe}$, and $\mathrm{Mn}$ atoms adsorbed on metallic surfaces and thin insulating films. Hirjibehedin et al. reported a large MAE for $\mathrm{Mn}$ adsorbed onto $\mathrm{CuN}$ surfaces assuming a vanishing orbital momentum $L=0$. This is surprising as the MAE results from the spin-orbit interaction in combination with the crystal-field splitting; it poses questions on a more profound interpretation of the experimental data. In contrast to the effective spin model, in XMCD measurements, a directiondependent expectation value $L$ of the orbital momentum is used to explain the MAE. ${ }^{10-12}$ These two models seem to contradict each other at first sight.

Here, we discuss the use of the effective spin model of single magnetic $3 d$ atoms in the case of a nonvanishing orbital magnetic moment, and show that in this case the effective spin
$\tilde{S}$ corresponds not to the real spin $S$ of the magnetic atom, but rather to the total angular momentum $J$ of the system. By including a larger number of states into the model, we come to a reinterpretation of experimental results. We show that by expressing the states of the atom and the change of the magnetization not in terms of $S$ but $J$ provides a better description of the underlying physics and unifies the aforementioned models.

\section{OUTLINE OF THE THEORY}

\section{A. Spin-orbit Hamiltonian}

We start with the electronic states of a free atom, which are given by solutions of the Dirac equation. The Hamiltonian comprises the pairwise electromagnetic interaction of all charged particles, i. e., the electron-nucleus interaction as well as the electron-electron interactions. As these interactions are relativistic, the spin-orbit interaction is included. The eigenstates of the atom consist of all possible $J$ multiplets obtained from coupling the spin and angular momentum of all the electrons. Thus, $J$ can vary considerably and, also, several multiplets with the same $J$ may exist.

A reasonable first guess for the atom's ground state can be obtained by Hund's rules. First, only open shells are considered. In the open shells, $S$ is maximized to minimize the exchange energy and $L$ is maximized to reduce the Coulomb energy. Finally, the total angular momentum $J=L+S$ should be at maximum for more than half-filled shells to minimize the spin-orbit interaction $\lambda \hat{\boldsymbol{L}} \hat{\boldsymbol{S}}$ ( $L S$ coupling).

The above rules are, however, approximations. A more general approach is to express the ground state as a multiplet of $2 J+1$ degenerate states. Each individual state is a mixture of states of the tensor product of all $L$ and $S$ multiplets of the electrons and, hence, can be written as

$$
\left|J m_{J}\right\rangle=\sum_{m_{L}, m_{S}} c_{m_{L}, m_{S}}\left|L m_{L}\right\rangle\left|S m_{S}\right\rangle,
$$

where $c_{m_{L}, m_{S}}$ are the mixing coefficients. The selection rules read as $L+S=J$ and $m_{L}+m_{S}=m_{J}$. The $m_{X}, X=L, S, J$, are the $z$ components of $\boldsymbol{L}, S$, and $\boldsymbol{J}$, respectively. Thus, when determining the expectation values for $L$ and $S$ of the 
ground state, irrational values may result. As a consequence, the magnetic spin and orbital moment may be irrational in units of Bohr's magneton (for an example of such a state, see Refs. 7 and 11).

\section{B. Crystal-field effects}

Second, we describe the electronic states of atoms that are deposited onto a substrate. The interaction with the substrate includes a crystal field, which modifies the center-symmetric potential of the free atom. This results in a mixture of the eigenstates of the free atom according to crystal-field theory. As the crystal field only acts on the spatial component of the wave function, the orbital momentum $L_{z}$ is partially quenched via the formation of $d_{x y}, d_{x z}$, etc., orbitals (for $d$ electrons). Their energetic splitting is determined by the symmetry and the size of the crystal field. In an extreme case, the crystal field may totally break Hund's rules, thus leading to low-spin states. Treating the states expressed in $L$ and $S$ in second-order perturbation theory, it turns out that the expectation value of $L$ depends on the direction of orbital momentum (Ref. 11). This implies that the orbital moment varies when the total angular moment is rotated by an external field. The variation of the orbital moment is often measured with XMCD and is related to the MAE.

Regarding the relativistic description, the crystal field mixes states of different $m_{J}$ and different $J$ multiplets. The resulting ensemble of states can still, however, be regarded as a set of multiplets, with only the lowest one populated at lower temperatures. One then describes this multiplet with the effective spin model by mapping the states on an effective $\tilde{S}$ multiplet with directional-dependent $g$ factors, which deviate from 2:

$$
\hat{H}_{\text {eff }}=E_{0}+E_{Z}+\sum_{n=2}^{\text {even }} \sum_{m=0}^{n} B_{n}^{m} \hat{O}_{n}^{m}+\sum_{n=2}^{\text {even }} \sum_{m=1}^{n} C_{n}^{m} \hat{\Omega}_{n}^{m} .
$$

Here, $E_{Z}$ denotes the Zeeman energy and $E_{0}$ the ground-state energy. The magnetic anisotropy is defined by additional terms in $\tilde{S}$, with $B_{n}^{m}$ and $C_{n}^{m}$ as the zero-field splitting coefficients. $\hat{O}_{n}^{m}$ and $\hat{\Omega}_{n}^{m}$ are Stevens operators of order $n$ of the components of the effective spin operator $\tilde{S}$. Due to time-reversal symmetry, only terms of even order are allowed. ${ }^{7,13}$ Further, depending on the symmetry of the crystal field, i.e. the atom's environment, some of the coefficients vanish. ${ }^{13}$ Due to spin algebra, $n \leqslant 2 \tilde{S}$. We would like to stress that, in the case of a nonzero $L$, the effective spin $\tilde{S}$ should be taken larger than the spin of the magnetic impurity. In the case wherein the mixing of the $J$ multiplets is not excessive, $\tilde{S}$ corresponds to the total angular moment $J$ of the lowest multiplet and, therefore, to $L+S$. This fact has been neglected in recent papers. ${ }^{2,3}$

In the case that some of the $2 J+1$ eigenstates have such large energies that they are not populated at sufficiently low temperatures, a subset of the $2 J+1$ states may be mapped onto an effective or better fictitious spin Hamiltonian. ${ }^{7}$ This explains the relative success of the models using a lower $\tilde{S}$. Such a description, however, requires a $g$ factor larger than 2 (see Ref. 14).

In the following, we use a simplified (or minimal) effective spin Hamiltonian, which takes into account only the first order of the Zeeman energy and omits those Stevens operators that are mixed products of the three (Cartesian) components of $\tilde{S}$ (Ref. 15) but keeps the Stevens operators that describe the lowest-order classical magnetic anisotropy. This approximation is valid for low magnetic fields (Zeeman energy smaller than level splitting) and crystal fields with high symmetry. This Hamiltonian reads as

$$
\hat{H}_{\mathrm{eff}}=\mu_{\mathrm{B}} \boldsymbol{H} \mathbf{g} \tilde{\boldsymbol{S}}+D \tilde{S}_{z}^{2}+\sum_{n=2}^{\text {even }} E_{n}\left(\tilde{S}_{+}^{n}+\tilde{S}_{-}^{n}\right) .
$$

Here, $\boldsymbol{H}$ denotes the magnetic field, $D$ induces a lowestorder uniaxial anisotropy along the $z$ direction (surface normal), while $E_{n}$ induces an $n$-fold anisotropy in the $x y$ plane (surface plane). The different coefficients $E_{n}$ have to be consistent with the symmetry of the crystal field to be nonvanishing. As the raising and lowering operators $\tilde{S}_{ \pm}$only operate within the $\tilde{S}$ multiplet, $n$ is limited to $n \leqslant 2 S$.

The quantum mechanical anisotropies are linked to the anisotropy of the orbital moment in the following way ${ }^{11}$ :

$$
D \propto \lambda^{2}\left(L_{\|}-L_{\perp}\right), \quad E \propto \lambda^{2}\left(L_{x}-L_{y}\right) .
$$

Note, however, that this is a simplified description assuming nondegenerated magnetic states. ${ }^{11}$

\section{Spin-flip scattering}

Third, we describe spin-flip scattering in the relativistic framework. In ITS experiments, the electron tunnels from the tip into the magnetic atom onto the surface. This scattering between the tunneling electron and the atom can be considered as electron-electron scattering. The leading term is the Coulomb interaction; spin-orbit coupling of the tunneling electron with the atom (Mott scattering) can be safely neglected because it is at least two orders of magnitude smaller than Coulomb scattering. We also neglect inelastic processes that do not alter the spin of the tunneling electron, such as electron-phonon interaction, as they are out of scope of this model.

The spin dependence of the scattering of the tunneling electron results from the Coulomb interaction in combination with the Pauli principle for the multielectron wave function. Thus, the overlap of the wave functions of the tunneling electron and the electrons of the atom depend on the spins of the involved electrons. The combined wave function can be split into the classical Coulomb energy and the exchange energy, which depends only on the spin of the electrons. This term is responsible for the transfer of the spin of the tunneling electron $s_{z}$ to the spin $m_{S}$ of the atom and can be written as ${ }^{16}$

$$
\hat{H}_{\mathrm{ex}}=J_{\mathrm{ex}}\left[\hat{s}_{z} \hat{S}_{z}+\frac{1}{2}\left(\hat{s}_{-} \hat{S}_{+}+\hat{s}_{+} \hat{S}_{-}\right)\right]
$$

where $J_{\text {ex }}$ is the exchange constant and $\hat{\boldsymbol{s}}$ as well as $\hat{\boldsymbol{S}}$ are the true spin operators of the electron and the atom, respectively. Note that the exchange interaction conserves the total spin of the system and describes the transfer of spin moment between the tunneling electron $(s=1 / 2)$ and the atom while the orbital momentum does not change. Thus, the action of the tunneling electrons on the atomic spin states obeys the selection rules $\Delta m_{S}= \pm 1,0$ and $\Delta m_{L}=0$. For a nonzero MAE, a spin flip requires energy, as was first studied by Heinrich et al. by ITS. ${ }^{17}$ 
When considering the relativistic eigenstates, these selection rules lead to $\Delta m_{J}=0, \pm 1$ as $m_{J}=m_{L}+m_{S}$. Thus, while spin-flip scattering only acts on the spin and not on the orbital momentum, it still leads to transitions within a $J$ multiplet; the latter may have a different multiplicity than the $S$ multiplet, but obeys the same selection rules. The same is true for $\tilde{S}$.

The spin-flip probability is only determined by the true spin and, consequently, the probability for inelastic excitations depends on the composition of the $J$ eigenstates in terms of $S$, namely, $c_{m_{L}, m_{S}}$ of Eq. (1). Therefore, although the excitation energies can be directly calculated from the effective spin Hamiltonian (3), the transition probabilities can not. To determine the latter exactly, the true wave functions of the eigenstates have to be known. However, a precise experimental measurement of the transition probabilities may be used to determine the $L-S$ composition of the eigenstates.

\section{A REVIEW OF RECENT EXPERIMENTS}

Finally, with the relativistic description of spin-flip scattering in mind, we review recent experiments on the MAE of single atoms.

\section{A. Co on $\operatorname{Pt}(111)$}

For single Co atoms on $\mathrm{Pt}(111)$, a MAE of $9.3 \mathrm{meV}$ was deduced by Gambardella et al. ${ }^{4}$ from XMCD measurements; a value of $10.3 \mathrm{meV}$ was obtained by Balashov et al. ${ }^{2}$ from ITS. Meier et al. also succeeded in measuring magnetization curves of single atoms using spin-polarized scanning tunneling spectroscopy (SP-STS). ${ }^{5}$ These three works use different models to describe the system: while Gambardella et al. and Meier et al. use classical spin (i.e., essentially, $\tilde{S} \rightarrow \infty$ ), Balashov et al. use the effective spin model with $\tilde{S}=1$. Neither of those is quite satisfactory due to the quantum mechanical nature of the system and to nonvanishing $L$ as determined by XMCD. ${ }^{4}$

Recent relativistic ab initio calculations of relaxed Co atoms on $\operatorname{Pt}(111)$ give estimates of $L$ and $S$ for this system. The total spin of the impurity located at a face-centered-cubic (fcc) site, including the induced spin in the closest $96 \mathrm{Pt}$ atoms, gives a magnetic spin moment of $2.35 \mu_{\mathrm{B}}$ and an orbital moment of $0.70 \mu_{\mathrm{B}}$ along the surface normal. A total magnetic moment of $3 \mu_{\mathrm{B}}$ is in good agreement with recent STM experiments. ${ }^{5}$ Thus, the total angular momentum of the embedded impurity is estimated to $J=S_{\mathrm{Co}}+S_{\mathrm{Pt}}+L_{\mathrm{Co}}+L_{\mathrm{Pt}}=1.875$. The same calculations performed for an atom on a hexagonal-closepacked (hcp) hollow site give $m_{S}=3.5 \mu_{\mathrm{B}}$ and $m_{L}=0.90 \mu_{\mathrm{B}}$.

Note that the $J$ of the Co impurity itself does not need to be a multiple of half integer, ${ }^{18,19}$ but when taking into account the full polarization cloud around the impurity, it necessarily is, as for the total angular momentum $\boldsymbol{J}_{\text {tot }}$ of the system including all electrons, the operator $J_{\text {tot }}^{2}$ commutes with the full system Hamiltonian. Thus, the $a b$ initio calculations suggest a total angular moment of $J=2$ or $3 / 2$ for fcc sites and $J=5 / 2$ for hcp sites.

Using this information, we can assume the following form for the Hamiltonian:

$$
\hat{H}_{\mathrm{Co} / \operatorname{Pt}(111)}=\mu_{\mathrm{B}} \boldsymbol{H g} \boldsymbol{J}+D J_{z}^{2} .
$$

Here, no in-plane term of sixth order for Co atoms is included as $2 J<6$. Additionally, the easy magnetization axis is out of plane $(D<0)$. Note that $\mathrm{g}$ and $D$ are, in principle, absorption site dependent. Unfortunately, neither XMCD nor ITS measurements provide site-specific information on the individual atoms, as the former is limited by resolution to an ensemble of atoms, and no data on atom positions were obtained in the latter. Ab initio calculations suggest, however, that most of the ITS measurements were performed on atoms on fcc sites. ${ }^{2}$

SP-STS studies also show that an additional indirect coupling is present between the atoms, strongly altering the perceived magnetic moment. ${ }^{20}$ This complicates the analysis of magnetization curves, especially the ones taken at $0.3 \mathrm{~K}$ and along the easy direction. This effect depends on the interatomic distance and is, therefore, most noticeable in the XMCD data $(\approx 2.3 \mathrm{~nm}$ between atoms), and least noticeable in the ITS data ( $\approx 5 \mathrm{~nm}$ between atoms). We can thus consider the energy difference between the magnetic ground state and the first excited state of $10.3 \mathrm{meV}$, as determined from ITS, to be unaffected by the indirect exchange.

First, we focus on SP-STS measurements that provide magnetization curves of single Co atoms on $\mathrm{Pt}(111)$ on fcc sites. ${ }^{5,21}$ To support our interpretation, we compare predictions of the magnetization curve using a quantum spin with the above values for $J$ and the MAE. For this, we calculated the state populations in an applied field along the $z$ axis for a single magnetic atom with $J=2, D=-3.4 \mathrm{meV}$ (Ref. 22), and varying the $g$ factor. The fitted magnetization curve of the quantum spin $\langle m\rangle$ [cf. the solid line in Fig. 1(a)] reproduces the measured magnetization curves excellently for $4.2 \mathrm{~K}$ and $g=1.36$, close to $g$ expected from the $a b$ initio calculations $[g=(L+2 S) /(L+S)=1.63]$. Note that the same set of parameters also nicely fits the magnetization curve recorded for the same atom at $0.3 \mathrm{~K}$ (not shown). Fitting the curves recorded at $0.3 \mathrm{~K}$ for other atoms requires a different $g$ due to the aforementioned indirect coupling between atoms. The case of $0.3 \mathrm{~K}$ is, however, not very informative as only the two ground states are thermally populated so that the calculated magnetization curves do not depend on the exact value of the MAE. We note that the magnetization curve fitted with $J=1$ and $g \leqslant 2$, corresponding to the effective spin model with $\tilde{S}=1$ (green dashed line), apparently deviates from the measured data, and that fits with $J>2$ lead to unphysical $g$ factors. A fit to the data with $J=3 / 2$ also reproduced the data well at $g=1.8$ (not shown).

Second, we discuss recent XMCD measurement of Co atoms on $\mathrm{Pt}(111)$, in which hard-axis and easy-axis magnetization curves of mixed ensembles of fcc and hcp atoms were recorded. The MAE and the magnetic moment, however, may depend on the adsorption site as suggested by ab initio calculations. $^{2}$ To obtain the MAE and the magnetic moment, a classical fitting neglecting the direction dependency of the $g$ factor was used in Ref. 4. To estimate this direction dependency, we use the change of $L$ with rotation from an easy to a hard direction. This change (which is responsible for the MAE) can be estimated from the Bruno model. ${ }^{10,12}$ Taking the above-mentioned MAE of $13.6 \mathrm{meV}, \Delta L$ is determined to $\approx 0.2$, in good agreement with the XMCD results. ${ }^{4}$ This 


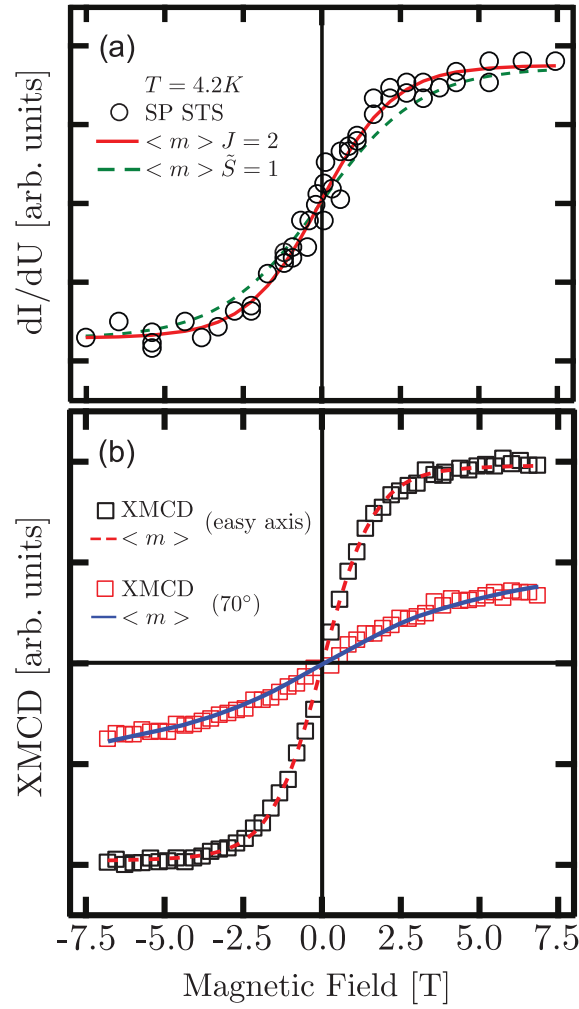

FIG. 1. (Color online) Comparison of magnetization curves of (a) SP-STS measurements (reproduced from Ref. 5; circles) and (b) XMCD measurements (reproduced from Ref. 4, squares) with calculations based on a quantum spin (solid and dashed lines).

translates into a difference between $g_{\text {hard }}$ and $g_{\text {easy }}$ of about 0.07. A magnetization curve can be calculated in an easy and a hard direction [cf. the red dashed and green solid lines in Fig. 1(b)] assuming this change in $g$, equally distributed absorption sites, and $J=2$ for fcc atoms and $J=4$ for hcp atoms using identical values of the anisotropy of $L$ for both sites, and fitting the out-of-plane $g$ factors (final values $g_{\text {fcc }}=1.64$ and $\left.g_{\text {hcp }}=1.74\right)$ and $D$ on hep cites. Note that fits with $J<4$ for hcp atoms lead to unphysical results, while fits with $J>4$ also resulted in good fits. This suggests that, indeed, either the magnetic moment of hcp atoms is larger than that of the fcc atoms or the indirect exchange in the XMCD measurements becomes sizable. The fitted $D$ value for hcp atoms is $-0.64 \mathrm{meV}$, leading to an MAE of $4.5 \mathrm{meV}$. This is in agreement with the tendency exposed by the $a b$ initio calculations, where the MAE for Co atoms on fcc sites is much larger than on hop sites.

In the case wherein the Hamiltonian (6) is extended to include the lowest-order in-plane anisotropy term as given by the Stevens operator $\hat{O}_{4}^{3}$, the available data are not sufficient to reliably determine all Hamiltonian parameters for atoms on both absorption sites. The attempts to fit the SP-STS and XMCD data with the extended Hamiltonian produced fits of the same quality, but with much larger errors, so that the calculated anisotropies did not differ within the error margin. To achieve a more accurate description, more information on Co atoms on hep absorption sites is required.
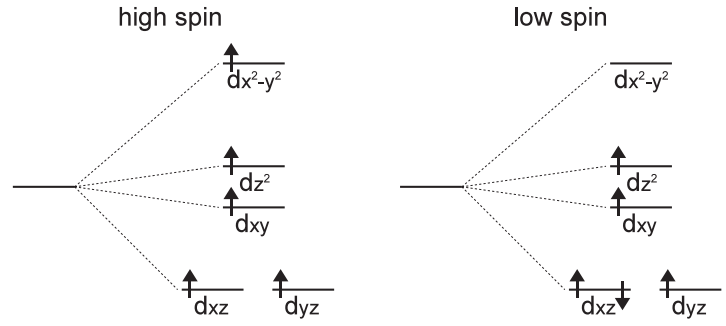

FIG. 2. Level scheme of $\mathrm{Mn}$ on $\mathrm{CuN}$ in the high- (left panel) and low-spin (right panel) configurations.

\section{B. Fe and Mn on $\mathrm{CuN}$}

As a final example, we review recent experiments by Hirjibehedin et al. for single $\mathrm{Mn}$ and $\mathrm{Fe}$ atoms on $\mathrm{CuN}^{3}$ in the relativistic picture. As the system has only a two-fold rotation axis, the effective spin Hamiltonian takes the form

$$
\hat{H}_{\mathrm{Fe} / \mathrm{CuN}}=\mu_{\mathrm{B}} \boldsymbol{H g} \boldsymbol{J}+D J_{z}^{2}+E\left(J_{+}^{2}+J_{-}^{2}\right),
$$

which coincides with the Hamiltonian used by Hirjibehedin et al.

For Mn atoms, one expects $S=5 / 2, L=0$, and $g=2$ from Hund's rules. In the presence of a crystal field of the Mn adsorption site (choosing the $x-y$ direction as the $\mathrm{N}-\mathrm{Mn}-\mathrm{N}$ bond direction), the $d$ levels split with degenerate $d_{x z}$ and $d_{y z}$ orbitals (see Fig. 2, left panel). This high-spin state $\Psi_{\mathrm{HS}}$ has $L=0$ and, thus, both the spin-orbit interaction and the MAE vanish. The $J=5 / 2$ high-spin multiplet can, however, mix with a second, excited $J=5 / 2$ multiplet in which the $d_{x^{2}-y^{2}}$ orbital is unoccupied (low-spin state $\Psi_{\mathrm{LS}}$ in Fig. 2). The low-spin case has a $L=1$ and $S=3 / 2$ and $g$ factor of 1.6.

In the experiments by Hirjibehedin et al., a $g$ factor of 1.9 was obtained from ITS measurements in high magnetic fields. This allows us to determine the mixing of the two

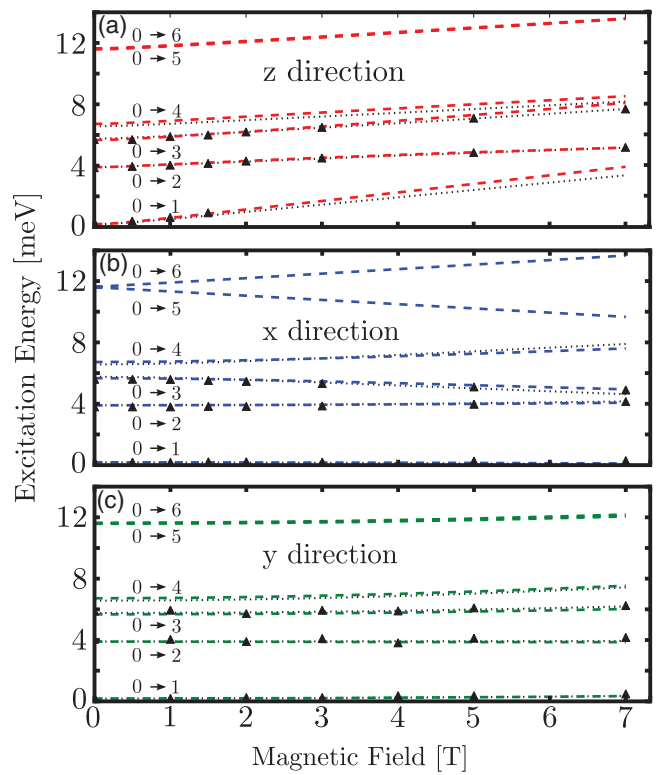

FIG. 3. (Color online) Excitation energies of $\mathrm{Fe}$ on $\mathrm{CuN}$ from the ground state " 0 " calculated with $J=3$ (dashed line) and with $\tilde{S}=2$ (dotted line) in the (a) $z$, (b) $x$, and (c) $y$ directions. The triangles show measured excitation energies (reproduced from Ref. 3 ). 
$J=5 / 2$ multiplets. The mixed state $\Psi_{\mathrm{M}}$ is a superposition of $\Psi_{\mathrm{HS}}$ and $\Psi_{\mathrm{LS}}$ with $\left|\Psi_{\mathrm{M}}\right|^{2}=3 / 4\left|\Psi_{\mathrm{HS}}\right|^{2}+1 / 4\left|\Psi_{\mathrm{LS}}\right|^{2}$. Thus, the anisotropy on $\mathrm{Mn}$ is entirely caused by the content of the low-spin state in the wave function.

For $\mathrm{Fe}$ on $\mathrm{CuN}$, however, a $g$ factor of 2.11 was estimated. At first sight, this cannot be explained by a relativistic model, as the contribution of $L$ to the total magnetic moment is with a $g$ factor of only 1 . In general, $g$ factors larger than 2 are observed if in the quantum-spin description only a subset of the full multiplet is considered, ${ }^{14}$ for example, if some of the high-energy states of the multiplet are not thermally populated.

With respect to the preceding, we examine the filling of the Fe $d$ orbitals in the presence of the same crystal field as for $\mathrm{Mn}$. The six electrons of the ground state would lead to $S=2$ and $L=1$ and thus to $J=3$, i. e., a multiplet larger than used in the recent work of Hirjibehedin et al. ${ }^{3}(\tilde{S}=2)$. By recalculating with $J=3$ and $g=1.67$ and comparing the calculated with the measured transitions, we find a good agreement for the transition energies $\Delta E$ for $D=-1.05 \mathrm{meV}$ and $E_{2}=0.42 \mathrm{meV}$ (cf. the dashed lines in Fig. 3). As the $J=3$ multiplet, in principle, allows for more transitions ${ }^{23}$ ("0" $\rightarrow$ "5" and "0" $\rightarrow$ "6"), we need to explain that these transitions have not been observed. A direct calculation of transition probabilities requires the exact compositions of the eigenstates, which are unknown; hence, we can only estimate an upper bound for those probabilities. We find that, similar to the transition " 0 " $\rightarrow$ " 4 " present in the fictitious spin-model calculations, but not observed in the spectra, ${ }^{3}$ the new transitions have probabilities below $1 \%$ and can thus be easily overlooked in ITS.

\section{CONCLUSION}

As a conclusion, the relativistic model combines the interpretations used to analyze XMCD data and ITS data. It is not in contrast to, but an extension and a unification of, the formerly used interpretations. A review of recent experiments shows a good agreement of the predictions of the model and the measurement. Using the relativistic model, some parameters including the MAE differ slightly from those extracted with the effective spin model or a classical model.

\section{ACKNOWLEDGMENTS}

W.W. acknowledges funding by the DFG (Wu 349/4-1) and the DAAD (50754493). C.C.K acknowledges support by the National Science Council of Taiwan (NSC 97-2112-M110-003-MY3) and the DAAD (100-2911-I-110-506). The authors acknowledge fruitful discussions with P. Gambardella, I. Mertig, A. Heinrich, S. Loth, B. Pilawa, and J. Wiebe.
${ }^{1}$ H. Brune and P. Gambardella, Surf. Sci. 603, 1812 (2009)

${ }^{2}$ T. Balashov, T. Schuh, A. F. Takács, A. Ernst, S. Ostanin, J. Henk, I. Mertig, P. Bruno, T. Miyamachi, S. Suga et al., Phys. Rev. Lett. 102, 257203 (2009).

${ }^{3}$ C. F. Hirjibehedin, C.-Y. Lin, A. F. Otte, M. Ternes, C. P. Lutz, B. A. Jones, and A. J. Heinrich, Science 317, 1199 (2007).

${ }^{4}$ P. Gambardella, S. Rusponi, M. Veronese, S. S. Dhesi, C. Grazioli, A. Dallmeyer, I. Cabria, R. Zeller, P. H. Dederichs, K. Kern et al., Science 300, 1130 (2003).

${ }^{5}$ F. Meier, L. Zhou, J. Wiebe, and R. Wiesendanger, Science 320, 82 (2008).

${ }^{6}$ C. Rudowicz, Magnetic Resonance Review 13, 1 (1987).

${ }^{7}$ C. Rudowicz and S. Misra, Applied Spectroscopy Reviews 36, 11 (2001).

${ }^{8}$ W. Wernsdorfer and R. Sessoli, Science 284, 133 (1999).

${ }^{9}$ D. Gatteschi, R. Sessoli, and J. Villain, Molecular Nanomagnets (Oxford University Press, New York, 2006).

${ }^{10}$ P. Bruno, Phys. Rev. B 39, 865 (1989).

${ }^{11}$ D. Dai, H. Xiang, and M.-H. Whangbo, J. Comput. Chem. 29, 2187 (2008).
${ }^{12}$ D. Weller, J. Stöhr, R. Nakajima, A. Carl, M. G. Samant, C. Chappert, R. Mégy, P. Beauvillain, P. Veillet, and G. A. Held, Phys. Rev. Lett. 75, 3752 (1995).

${ }^{13}$ S. Misra, C. P. Poole, and H. Farach, Appl. Magn. Reson. 11, 29 (1996).

${ }^{14} \mathrm{~A}$. Abragam and B. Bleaney, Electron Paramagnetic Resonance of Transition Ions (Clarendon, Oxford, 1970).

${ }^{15}$ C. Romeike, M. R. Wegewijs, W. Hofstetter, and H. Schoeller, Phys. Rev. Lett. 96, 196601 (2006).

${ }^{16}$ J. Fransson, Nano Lett. 9, 2414 (2009).

${ }^{17}$ A. J. Heinrich, J. A. Gupta, C. P. Lutz, and D. M. Eigler, Science 306, 466 (2004).

${ }^{18}$ P. Anderson, Phys. Rev. 124, 41 (1961).

${ }^{19}$ A. A. Khajetoorians, S. Lounis, B. Chilian, A. T. Costa, L. Zhou, D. L. Mills, J. Wiebe, and R. Wiesendanger, Phys. Rev. Lett. 106, 037205 (2011)

${ }^{20}$ L. Zhou, J. Wiebe, S. Lounis, E. Vedmedenko, F. Meier, S. Blügel, P. Dedrichs, and R. Wiesendanger, Nat. Phys. 6, 187 (2010).

${ }^{21} \mathrm{~J}$. Wiebe (private communication).

${ }^{22} E_{s f}=D(2 J-1)$ with the spin-flip energy from the ground state to the first excited state $E_{s f}=10.3 \mathrm{meV}$.

${ }^{23}$ Numbers are taken to name the states by energies at $0 \mathrm{~T}$, starting with the ground state 0 . 